\title{
Evaluation of the antioxidant properties of fruit and flavoured black teas
}

\author{
Anna Pękal · Paulina Dróżdż $\cdot$ Magdalena Biesaga • \\ Krystyna Pyrzynska
}

Received: 4 November 2010/Accepted: 17 February 2011/Published online: 1 March 2011

(C) The Author(s) 2011. This article is published with open access at Springerlink.com

\begin{abstract}
Objective Antioxidant properties of the water extracts of the commercial bagged fruit and flavoured black teas were evaluated and compared with typical black teas of C. sinensis.

Methods Folin-Ciocalteu (FC) assay, cupric ion reducing antioxidant capacity (CUPRAC) and DPPH radical method were used for these purposes. The content of selected flavonoids and phenolic acids was also determined by highperformance liquid chromatography with tandem mass spectrometry in the negative electrospray ionization mode. Results Flavoured black teas contain significantly higher level of catechins, quercetin, and rutin, while the content of chlorogenic and caffeic acids as well as naringin and hesperidin was higher in fruit teas. Supplementation with these flavonoids could reduce blood glucose. In FC and DPPH assays, the antioxidant properties of studied tea infusion increases in the order: fruit tea $<$ flavoured black tea $>$ premium black tea, while in CUPRAC method, some aromatized teas exhibit the highest antioxidant properties. Tea infusions with nice smell of fruits would also support the human diet with some source of antioxidants.
\end{abstract}

Keywords Fruit and flavoured teas - Antioxidant properties · Polyphenol content · CUPRAC $\cdot$ DPPH

\section{Introduction}

Tea is one of the most widely consumed beverages in the world, next only to water. It is produced from the same

A. Pękal · P. Dróżdż · M. Biesaga · K. Pyrzynska ( $($ )

Department of Chemistry, University of Warsaw,

Pasteura 1, 02-093 Warsaw, Poland

e-mail: kryspyrz@chem.uw.edu.pl plant Camelia sinesis and can be categorized into 3 types, depending on the level of fermentation, i.e., green (unfermented), oolong (partially fermented), and black (fermented) tea. Although this process is often assumed, incorrectly, to be fermentation, which usually implies additives, the more correct term should be oxidation, which means exposure to air while drying [1]. Green tea processing includes a step to inactive oxidizing enzymes, such as polyphenol oxidase, which partially converts monomeric polyphenols (mainly catechins) into dimeric/oligomeric compounds, such as theaflavins and thearubigins, responsible for the characteristic aroma and colour of black tea. Oolong tea is a result of oxidation being stopped somewhere in between that of green and black tea and therefore contains flavonoids that are found in both teas. Not only green and black tea but also various kinds of fruit and flavoured teas are very popular in many European countries. These teas are obtained by the addition of natural aromas and dry fruits or herbs to tea leaves in the last stage of processing before packing. They are popular because of their fragrance, therapeutic applications, and lower content of caffeine, which could inhibit calcium absorption, particularly for older people $[2,3]$. One cup of very strong brewed tea (about $45 \mathrm{mg}$ of caffeine) might lead to a loss of 2-3 mg of calcium [4].

Tea polyphenols, especially flavonoids, have attracted considerable interest because of their associated health properties, and these have been summarized in several papers [5-7]. The studies have demonstrated that tea flavonoids possess strong antioxidant activity and metalchelating properties, thus may protect cells and tissues against reactive oxygen and nitrogen species. A typical cup of green tea of usual strength $(1.5 \% \mathrm{w} / \mathrm{v})$ contains an amount of antioxidant power similar to that found in 100-200 mg of pure vitamin C) [8]. Regular intake of tea 
may therefore improve antioxidant status in vivo and help lower risk of certain types of cancer and coronary heart disease [9].

Considering the increasing interest in the health properties of tea, the present study has been designed to compare the antioxidant properties of different commercially available bagged flavoured black teas and fruit teas (all Lipton brand names). The typical black teas of $C$. sinensis (Yellow label) were used as positive control. Like most branded teas, Lipton teas are a blend selected from many different plantations around the world, from well-known producing countries like China, India and Sri Lanka. FolinCiocalteu (FC) assay, cupric ion reducing antioxidant capacity (CUPRAC), and DPPH radical method were used to evaluate the antioxidant properties of tea infusions. The content of selected flavonoids and phenolic acids was also determined by LC-MS in the negative electrospray ionization mode.

\section{Materials and methods}

\section{Reagents and apparatus}

The commercial standards of flavonoids and polyphenolic acids, Folin-Ciocalteu's reagent, and DPPH (2,2-diphenyl1-picrylhydrazyl radicals) as well as the rest of the chemicals were purchased from Sigma (Steinheim, Germany). Methanol and acetonitrile were of HPLC gradient grade from Merck (Darmstadt, Germany). Ultrapure water from Milli-Q system (Millipore, Bedford, MA, USA) with a conductivity of $18 \mathrm{MQ}$ was used in all experiments. All solutions were filtered through $0.45-\mu \mathrm{m}$ membranes (Millipore) and degassed prior to use.

Spectrophotometric determinations were carried out on a Perkin Elmer model Lambda 20 UV-VIS spectrophotometer with cuvettes of $1 \mathrm{~cm}$ length. Spectra were recorded in the range from 220 to $600 \mathrm{~nm}$ with $0.2-\mathrm{nm}$ resolution. Data were processed with WinLab software.

Chromatographic analysis was performed with a Shimadzu LC system consisted of binary pumps LC20-AD, degasser DGU-20A5, column oven CTO-20AC, autosampler SIL-20AC, detector UV SPD 20A connected to 3200 QTRAP Mass spectrometer (Applied Biosystem/MDS SCIEX). A MS system equipped with electrospray ionization source (ESI) operated in negative ion mode and a quadrupole mass analyser in a scan mode from 50 to $1500 \mathrm{~m} / \mathrm{z}$. ESI conditions were the following: capillary temperature $450{ }^{\circ} \mathrm{C}$, curtain gas at $0.3 \mathrm{MPa}$, auxiliary gas at $0.3 \mathrm{MPa}$, negative ionization mode source voltage$4.5 \mathrm{kV}$. Nitrogen was used as curtain and auxiliary gas.
Compounds were separated on Kinetex ${ }^{\mathrm{TM}}$ (Phenomenex) C-18 column $(100 \times 2.1 \mathrm{~mm}, 2.6 \mu \mathrm{m})$ with precolumn at $30{ }^{\circ} \mathrm{C} ; 2 \mathrm{mM}$ formic acid $(\mathrm{pH} 2.8)$ as eluent $\mathrm{A}$ and acetonitrile as eluent $\mathrm{B}$ were used. The mobile phase was delivered at $0.2 \mathrm{~mL} / \mathrm{min}$ in linear gradient mode: $0-5 \mathrm{~min}$ $20 \%$ B, $10-15 \min 25 \%$ B, 20-25 min 30\% B, 30-31 min $90 \%$ B, $32 \min 20 \%$ B. Compounds were identified by comparing retention time and $\mathrm{m} / \mathrm{z}$ values obtained by MS and $\mathrm{MS}^{2}$ with the mass spectra from standards tested under the same conditions (Table 1). Quantification of compounds was made from the calibration curves obtained in multiple reaction mode (MRM) [10].

Teas and preparation of infusions

All bagged teas were purchased from a local market. They contain, except leaves of $C$. sinensis, also dry fruit and herbs which are visible, as well as some natural aroma. This set of teas was chosen as they are very popular and widely sold. This includes four fruit teas with commercial names: Caribbean (containing orange leaves, rosehip, papaya, hibiscus), Delight citrus (rosehip, apple, lemon, and grapefruit peels), Char Ming (blackcurrant, rosehip, hibiscus), and Temptation (rosehip, strawberry, blackberry, raspberry) as well as four flavoured black teas named: Blue fruit (bilberry, blackcurrant, blackberry, raspberry), Forest fruit (raspberry, cherry, blackberry, redcurrant), Citrus (orange, lemon, grapefruit, limon), and Tropical fruit (pineapple, grapefruit peels). For comparison, also typical black tea Lipton brand (Yellow label) was studied. The content of tea bags supplied from the market was opened, and tea was weighted; masses were ranging between 1.64 and $2.02 \mathrm{~g}$.

The tea bags were dipped into $200 \mathrm{~mL}$ of freshly boiled water for $5 \mathrm{~min}$ to represent the typical quantity consumed by tea drinkers. After the infusion time, the bags were removed and the partly turbid solutions were filtered after cooling to room temperature and then analysed.

Antioxidant properties of teas

In Folin-Ciocalteu assay, $1 \mathrm{~mL}$ of tea infusion was introduced into test tubes followed by $0.1 \mathrm{~mL}$ of $\mathrm{FC}$ reagent and $0.9 \mathrm{~mL}$ of water. The tubes were allowed to stand for $5 \mathrm{~min}$. At the end of this period, $1 \mathrm{~mL}$ of sodium carbonate $(7 \%, \mathrm{w} / \mathrm{v})$ and $0.4 \mathrm{~mL}$ of water were added, and 10 more min was allowed for stabilization of the blue colour formed. The absorbance against a reagent blank was measured at $765 \mathrm{~nm}$. The results were expressed as gallic acid equivalent (GAE) in $\mathrm{mg} / \mathrm{g}$ of dry matter. The determinations were carried out for three independently prepared samples of each type of tea. 
Table 1 LC/MS/MS

characteristics of studied compounds in negative ion mode

\begin{tabular}{lcllll}
\hline Compound & Retention time, min & Q1 Mass (amu) & Q3 Mass (amu) & DP, V & CE, V \\
\hline Gallic acid & 2.18 & 169 & 125 & -45 & -20 \\
Chlorogenic acid & 2.39 & 353 & 191 & -20 & -22 \\
Caffeic acid & 2.98 & 179 & 135 & -10 & -24 \\
Syringic acid & 3.02 & 197 & 182 & -20 & -18 \\
Rutin & 3.55 & 609 & 300 & -65 & -56 \\
p-coumaric & 4.14 & 163 & 119 & -20 & -18 \\
Synapic acid & 4.48 & 223 & 164 & -35 & -20 \\
Ferulic acid & 4.75 & 193 & 134 & -30 & -20 \\
Catechin & 5.12 & 289 & 109 & -45 & -34 \\
Naringin & 5.49 & 579 & 271 & -80 & -52 \\
Hesperidin & 5.75 & 609 & 301 & -65 & -30 \\
Epicatechin & 8.20 & 289 & 109 & -40 & -32 \\
Epigallocatechin 3-gallate & 9.55 & 457 & 169 & -35 & -22 \\
Trans-cinnamic acid & 13.26 & 146 & 103 & -30 & -14 \\
Luteolin & 14.46 & 285 & 133 & -60 & -44 \\
Quercetin & 14.72 & 301 & 151 & -40 & -30 \\
Naringenin & 18.71 & 271 & 151 & -45 & -26 \\
Genistein & 19.41 & 269 & 133 & -75 & -52 \\
Apigenin & 20.62 & 269 & 117 & -55 & -42 \\
Kaempferol & 21.82 & 285 & 151 & -45 & -25 \\
Isorhamnetin & 28.86 & 315 & 300 & -35 & -24 \\
Rhamnetin & 30.88 & & 165 & -30 \\
\hline & & 315 & & -35 \\
\hline
\end{tabular}

Radical scavenging activity was assessed using the 2,2-diphenyl-1-picrylhydrazyl (DPPH) assay; $0.1 \mathrm{~mL}$ of tea infusion was mixed with $2.4 \mathrm{~mL}$ DPPH solution $\left(3 \times 10^{-5} \mathrm{M}\right)$ in methanol, and immediately, the change of absorbance at $539 \mathrm{~nm}$ was recorded over time against the blank. The results were expressed as the percentage of reduction (inhibition) of the DPPH according to expression: $\left(A_{0}-A_{\mathrm{t}}\right) / A_{\mathrm{o}} \times 100$, where $A_{\mathrm{o}}$ is the initial absorbance, and $A_{\mathrm{t}}$ is the absorbance at increasing time $t$.

For assessing cupric ion reducing ability (CUPRAC), the assay described by Apak et al. [11] was adapted. In a test tube, $1 \mathrm{~mL} \mathrm{CuCl}_{2}$ solution $\left(1.0 \times 10^{-2} \mathrm{M}\right)$ was mixed with $1 \mathrm{~mL}$ of neocuproine alcoholic solution $\left(7.5 \times 10^{-3} \mathrm{M}\right)$ and $1 \mathrm{~mL}$ of $1 \mathrm{M} \mathrm{NH} \mathrm{NH}_{4} \mathrm{AC}$ buffer $(\mathrm{pH}$ 7), followed by mixing $0.5 \mathrm{~mL}$ of tea infusion and $0.6 \mathrm{~mL}$ of water. Absorbance against the reagent blank was measured after $30 \mathrm{~min}$ at $450 \mathrm{~nm}$. In another sets of experiments, the tube containing sample and reagents was incubated in a water bath at a temperature of $50{ }^{\circ} \mathrm{C}$ for $20 \mathrm{~min}$. Then the tube was cooled under running water, and its $A_{450}$ value was measured. The infusions of black teas were diluted tenfold with water to keep the absorbance at $450 \mathrm{~nm}$ between 0.2 and 0.4 absorbance units. The calibration curve was built-up with trolox (TR), and the antioxidant activity of the tea infusions was expressed as trolox equivalent (mmol TR/g of dry matter).

\section{Results}

HPLC-MS analysis

Examples of the chromatograms of tea infusions by LC/ MS/MS are presented in Fig. 1. Peak identity was established by both the retention time compared with that of the standards and the characteristic transitions (precursor and product ion pair). The concentration of some relevant flavonoids and phenolic acids found in the selected tea infusions are presented in Table 2. Gallic, chlorogenic, $p$-coumaric, ferulic and synaptic acid were detected in all studied infusions. Flavoured black tea infusions had significant content of gallic acid (5.59-8.51 mg/L) but did not contain synaptic acid (except detected traces in Citrus and Blue fruit teas). The level of chlorogenic and caffeic acid was higher in fruit teas. In plants, caffeic acid is formed from $p$-coumaric acid and is transformed later to ferulic acid. The order of hydroxycinnamic acids found in citrus fruits was $p$-coumaric acid $>$ ferulic acid $>$ caffeic acid [12]. This order agrees with the content of these compounds in the studied fruit teas which contain orange leaves (Caribbean) and citrus peels (Delight citrus). Also these phenolic acids were detected in similar order in black tea aromatized with citrus fruits (Citrus) (Table 2). No peaks of $p$-HPA and trans-cinnamic acid (except Caribbean tea) 
were recorded, which confirms data obtained by Mattila et al. [13].

In relation to the catechin content in the analysed samples, flavoured black teas contain much higher level of these compounds in comparison with fruit black teas and even to premium black tea (Yellow label). The level of epigallocatechin 3-gallate (EGCG), polyphenol with very high antioxidant activity [14], ranged from 12.3 to $29.8 \mathrm{mg} / \mathrm{L}$ in studied flavoured teas. In some fruit tea infusions, epicatechin (Char Ming) and EGCG (Char Ming and Temptation) were not found.

The predominant flavonones in citrus fruits are naringenin and hesperidin [15], and these compounds were detected in studied fruit teas. Particularly, Delight citrus tea is very rich in these flavonones and contains 5.81 and $25.2 \mathrm{mg} / \mathrm{L}$ of hesperidin and naringin, respectively. Flavone luteolin was detected only in brewed black teas in the range of $0.006-0.010 \mathrm{mg} / \mathrm{L}$. Amongst flavonols, quercetin and traces of myricetin (about $0.014 \mathrm{mg} / \mathrm{L}$ ) were present only in black tea infusions. Kaempferol as aglycone has not been found in all studied teas, although its presence in the forms of different glycosides was determined in several tea and herbal infusions [16]. The concentration of rutin was much higher in teas of $C$. sinensis than in fruit teas; the highest value of $18.75 \mathrm{mg} / \mathrm{L}$ was found in Yellow label brand. Differences in flavonoid content per teas serving are the result of tea variety, weight of tea used, and to a lesser extent, brewing technique [17]. It should be mentioned that some flavoured teas, particularly Blue fruit and Forest fruit, contain also anthocyanins. Apart from their colouring effects in fruits, anthocyanins show ability to prevent lipid oxidation and scavenging activity against various free radicals [18].

\section{Antioxidant properties}

The results obtained by Folin-Ciocalteu method varied widely in the studied types of tea. The obtained results for fruit tea infusions are very similar (in the range of 123-141 mg GAE/g dry weight) but much lower than for favoured black teas (Fig. 2). The highest results were found for Blue fruit (361.6 mg GAE/g), while the lowest for Caribbean (122.9 mg GAE/g). Typical Lipton black tea (Yellow label brand) exhibits the antioxidant properties equals to $420 \mathrm{mg} \mathrm{GAE} / \mathrm{g}$ and $835.6 \mathrm{mg} \mathrm{GAE} / 200 \mathrm{~mL}$ of infusion.

Two set of experiments were conducted using CUPRAC method; in the first assay, absorbance was measured after keeping the mixed components at room temperature for $30 \mathrm{~min}$, while in the second assay, the samples were incubated after reagent addition at $50{ }^{\circ} \mathrm{C}$. The obtained results were expressed in mmol of $\mathrm{TR} / \mathrm{g}$ of tea as trolox equivalent (TE) and are presented in Fig. 3. As expected, black tea infusion exhibit higher TE values than studied fruit teas. These teas contain much higher content of quick reacting flavonoids such as catechins, quercetin and gallic acid, which have also the highest antioxidant capacities in CUPRAC assay [11]. Slow reacting antioxidants such as naringenin, sinapic and chlorogenic acids, the most abundant phenolic in the citrus and berry families as well as in some other fruits, needed elevated temperature incubation to complete their oxidation with the copper(II)-neocuproine reagent. In the measurement without incubation, the highest antioxidant capacity of $10.8 \mathrm{mg} \mathrm{TR} / \mathrm{g}$ was observed for Forest Fruit tea, which is aromatized mainly with raspberry and blackberry. After incubation, all TE values increased, amongst fruit teas particularly for Char Ming and Temptation (fruit teas) as well as for Tropical fruit (black flavoured tea).

The percentages of DPPH radical scavenging effect of each tea infusion for 5 and 20 min after addition of reagent are shown in Table 3. As can be seen, black flavoured teas as well as typical black teas of $C$. sinensis (Yellow label) exhibit high capacity to scavenging the DPPH radicals already in $5 \mathrm{~min}$. Very similar inhibition of DPPH in the range of 59-68\% was observed for that kind of teas, despite the time after mixing the components. The decay slope and the absorbance level reached by the remaining DPPH radicals vary significantly with different types of studied tea infusions (Fig. 4). A fast and remarkable decrease in the absorbance of DPPH was observed during the first few minutes of the reaction for black teas, followed by slow subsequent disappearance of the reagent. This fast step essentially refers to the electron transfer process from $\mathrm{B}$ ring $\left(3^{\prime}-\mathrm{OH}\right.$ and $\left.4^{\prime}-\mathrm{OH}\right)$ of flavonoid molecules to DPPH and latter kinetic reflects the remaining activity of the oxidation-degradation products [19]. For flavoured black teas, constant slow decay of DPPH absorbance was observed. The higher antioxidant properties of black teas could be due to the content and composition of major polyphenols (Table 2).

\section{Discussion}

Tea provides a dietary source of biologically active compounds, including polyphenols, which help prevent a wide variety of diseases. The antioxidant activity of tea polyphenols is mainly due to their ability to scavenge free radicals and to increased activity of some detoxifying enzymes in small intestine, liver and lungs [2]. Thus, tea consumed throughout the world is believed to be not only a popular beverage but also an antioxidant agent available in everyday life. Flavoured and fruit teas, which are popular because of their fragrance and lower amounts of caffeine, could be a good source of compounds with antioxidant 
Fig. 1 Total ion current of MRM of a Delight citrus and b Tropical fruit infusions and traces of phenolic compounds in MRM mode. MS/MS conditions described in the text

\section{(A) Delight citrus}

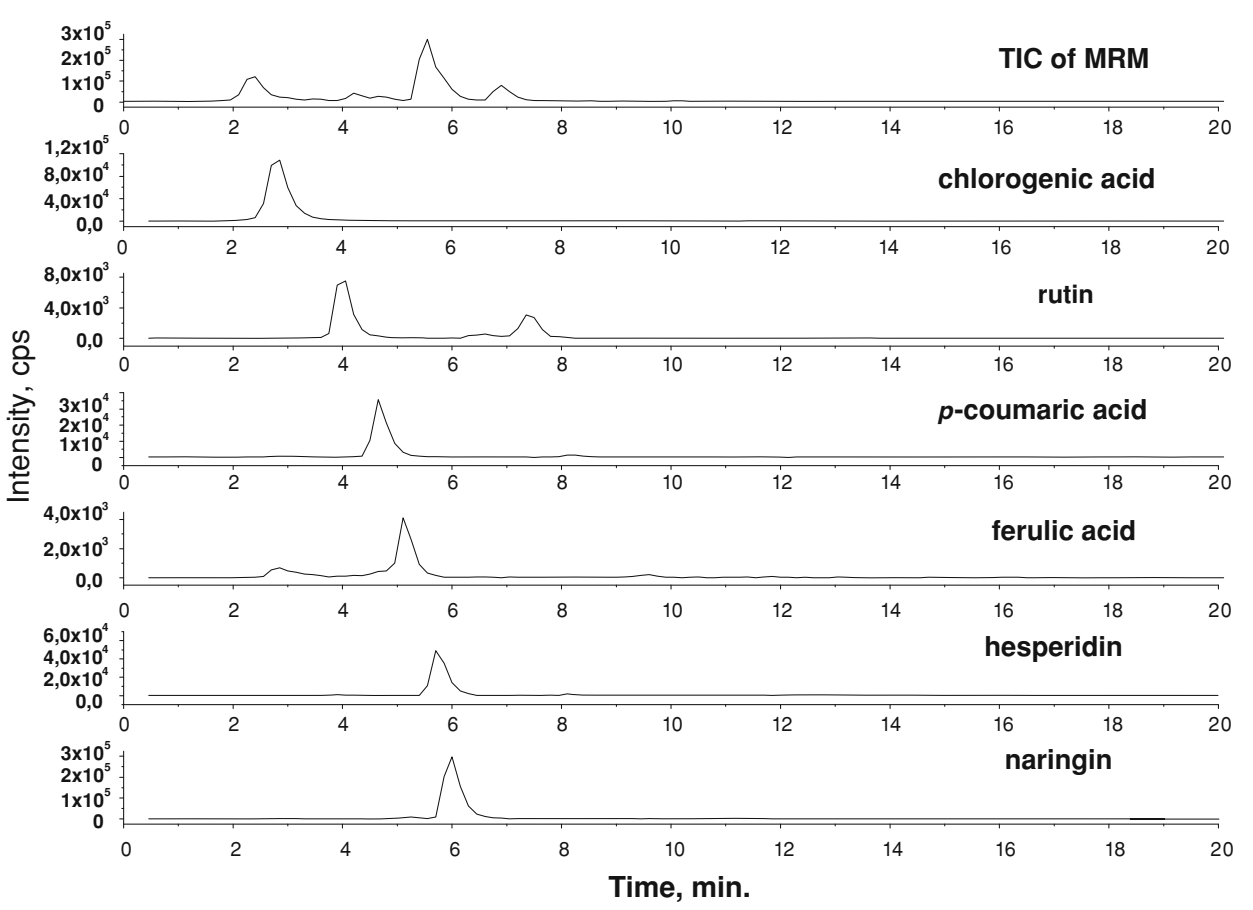

\section{(B) Tropical fruit}
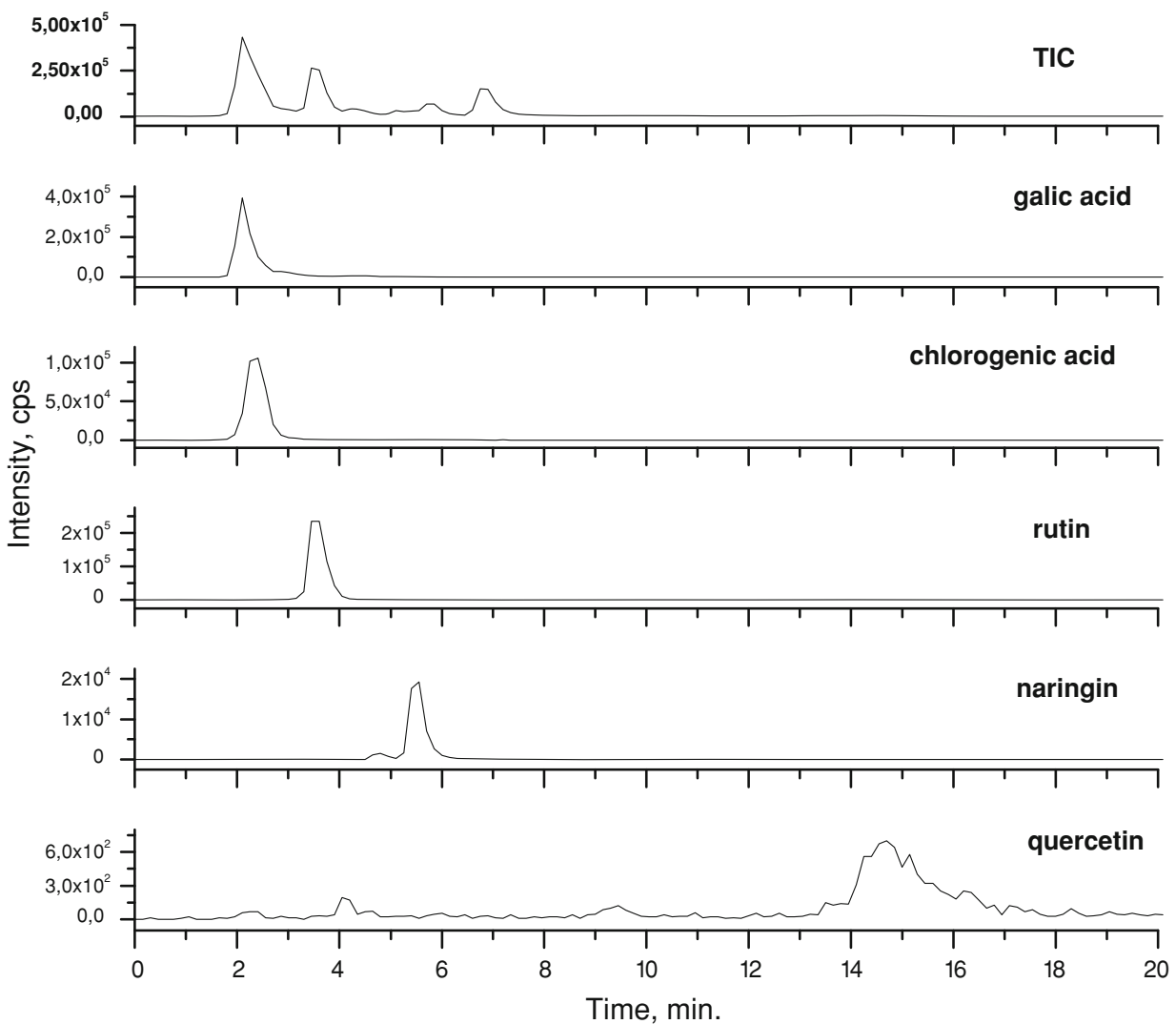
Table 2 Contents of selected flavonoids and phenolic acids in tea infusions expressed in $\mathrm{mg} / \mathrm{L}$

\begin{tabular}{|c|c|c|c|c|c|c|c|c|c|c|}
\hline & Caribbean & $\begin{array}{l}\text { Delight } \\
\text { citrus }\end{array}$ & $\begin{array}{l}\text { Char } \\
\text { ming }\end{array}$ & Temptation & $\begin{array}{l}\text { Blue } \\
\text { fruits }\end{array}$ & $\begin{array}{l}\text { Forest } \\
\text { fruits }\end{array}$ & Citrus & $\begin{array}{l}\text { Tropical } \\
\text { fruits }\end{array}$ & $\begin{array}{l}\text { Yellow } \\
\text { label }\end{array}$ & $\begin{array}{l}\mathrm{LOD} \\
(\mathrm{mg} / \mathrm{L})\end{array}$ \\
\hline Rutin & 0.18 & 0.37 & 1.89 & 1.16 & 9.91 & 9.05 & 8.59 & 11.3 & 18.8 & 0.001 \\
\hline Quercetin & nd & nd & nd & nd & 0.10 & 0.06 & 0.04 & 0.3 & 0.17 & 0.03 \\
\hline Naringin & 4.99 & 25.2 & nd & nd & 0.007 & 0.009 & 3.51 & 1.64 & nd & 0.001 \\
\hline Rhamnetin & 0.005 & 0.004 & 0.004 & 0.004 & 0.006 & 0.038 & 0.002 & 0.004 & 0.003 & 0.001 \\
\hline Luteolin & nd & nd & nd & nd & 0.008 & nd & 0.006 & 0.010 & 0.009 & 0.001 \\
\hline Hesperidin & 0.23 & 5.81 & nd & nd & nd & nd & 0.69 & nd & nd & 0.005 \\
\hline Catechin & 1.58 & 0.61 & 0.55 & 0.88 & 12.0 & 7.97 & 9.17 & 15.9 & 1.71 & 0.001 \\
\hline Epicatechin & 1.87 & 0.72 & nd & 1.04 & 7.01 & 9.42 & 10.9 & 18.8 & 2.35 & 0.05 \\
\hline $\begin{array}{l}\text { Epigallocatechin } \\
\text { 3-gallate }\end{array}$ & 0.63 & 0.024 & nd & nd & 12.3 & 14.9 & 21.7 & 29.8 & 9.47 & 0.001 \\
\hline Gallic acid & 0.049 & 0.051 & 0.031 & 0.007 & 6.32 & 5.59 & 7.94 & 8.51 & 8.48 & 0.0005 \\
\hline Ferulic acid & 0.10 & 0.10 & 0.032 & 0.021 & 0.025 & 0.025 & 0.0060 & 0.036 & 0.046 & 0.001 \\
\hline Synapic acid & 0.031 & 0.065 & 0.050 & 0.037 & 0.001 & nd & 0.010 & nd & nd & 0.001 \\
\hline Caffeic acid & 0.041 & 0.052 & 0.076 & 0.037 & 0.018 & 0.020 & 0.031 & 0.023 & 0.020 & 0.001 \\
\hline Chlorogenic acid & 2.44 & 1.74 & 2.72 & 2.44 & 1.77 & 1.72 & 1.60 & 1.72 & 1.93 & 0.001 \\
\hline$p$-coumaric acid & 0.32 & 0.24 & 0.069 & 0.098 & 0.18 & 0.22 & 0.36 & 0.27 & 0.49 & 0.0005 \\
\hline
\end{tabular}

$n d$ not detected, e.g. $<$ LOD

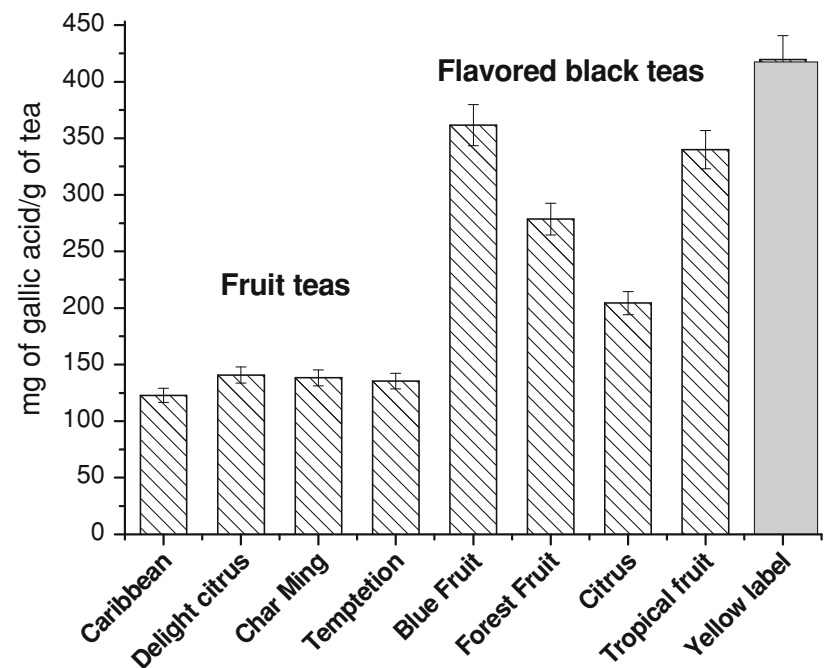

Fig. 2 Antioxidant capacity of flavoured black and fruit tea infusions measured by Folin-Ciocalteu method

properties. Moreover, they are characterized by the lesser astringent and bitter taste perceived upon black tea consumption. Bitterness of tea is generally ascribed to the combination of catechins, saponins, polyphenols and amino acids [20, 21]. Some flavonoids are very bitter whereas others not, depending on the type of the glycoside chain. Naringin and neohesperidin are very bitter, whereas hesperidin is tasteless [20].

These studies show very marked differences in the level of some major flavonoids and phenolic acids between flavoured black tea and fruit teas. The last type contains,

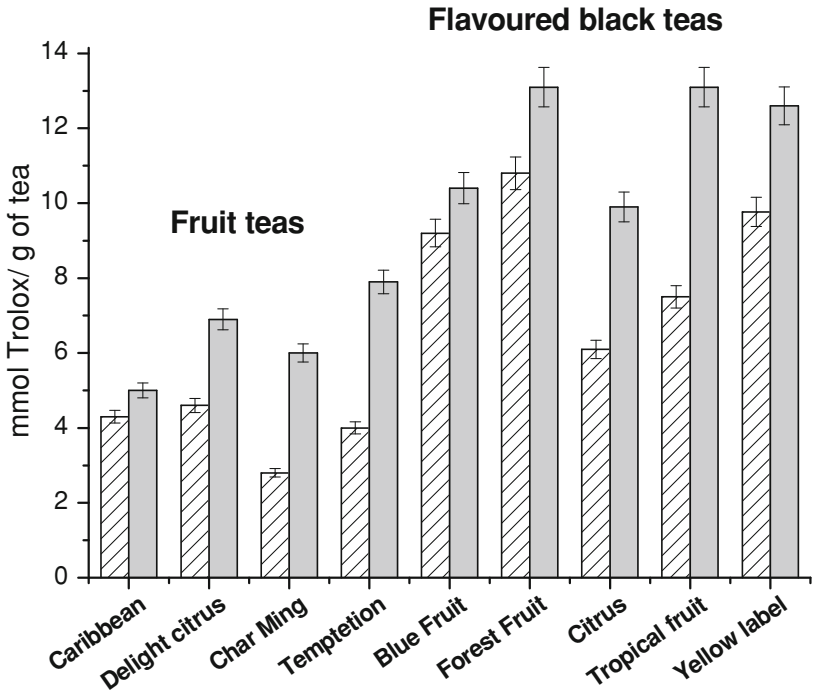

Fig. 3 Antioxidant capacities of tea infusions (expressed as mmol $\mathrm{TR} / \mathrm{g}$ ) measured by the CUPRAC method; lined rectangle-without incubation, shaded rectangle-incubation at $50{ }^{\circ} \mathrm{C}$

except leaves of $C$. sinensis, also dry fruit and herbs, which are visible, while natural aroma of different fruits is added to fermented tea leaves for producing flavoured products. Fruit teas are richer in phenolic acids, particularly chlorogenic, ferulic and caffeic acid. The research studies suggest that chlorogenic acid could help lower blood sugar levels and control blood glucose levels in type II diabetes mellitus condition [22, 23]. Caffeic acid functions as a carcinogenic inhibitor and has tumour-shrinking properties [22]. HPLC analysis of Delight citrus and Caribbean teas showed the 
Table 3 Scavenging effect of aqueous extracts from selected teas on DPPH radicals

\begin{tabular}{lll}
\hline Tea & \multicolumn{2}{l}{ Scavenging effect, $\%^{\mathrm{a}}$} \\
\cline { 2 - 3 } & $5 \mathrm{~min}$ & $20 \mathrm{~min}$ \\
\hline Caribbean & $33.5 \pm 1.7$ & $51.9 \pm 2.4$ \\
Delight citrus & $25.1 \pm 1.2$ & $39.6 \pm 1.9$ \\
Char Ming & $29.1 \pm 1.4$ & $40.3 \pm 2.0$ \\
Temptation & $32.1 \pm 1.6$ & $55.0 \pm 2.2$ \\
Blue fruit & $67.6 \pm 3.3$ & $68.4 \pm 3.4$ \\
Forest fruit & $63.4 \pm 3.1$ & $65.6 \pm 3.3$ \\
Citrus & $58.6 \pm 2.9$ & $58.5 \pm 2.9$ \\
Tropical fruit & $64.5 \pm 3.1$ & $67.7 \pm 3.2$ \\
Lipton yellow label & $63.3 \pm 3.0$ & $63.6 \pm 3.0$ \\
\hline
\end{tabular}

${ }^{a}$ Scavenging effect calculated as $\left(A_{0}-A_{\mathrm{t}}\right) / A_{\mathrm{o}} \times 100$, where $A_{\mathrm{o}}$ is the initial absorbance and $A_{\mathrm{t}}$ is the absorbance at increasing time $t$

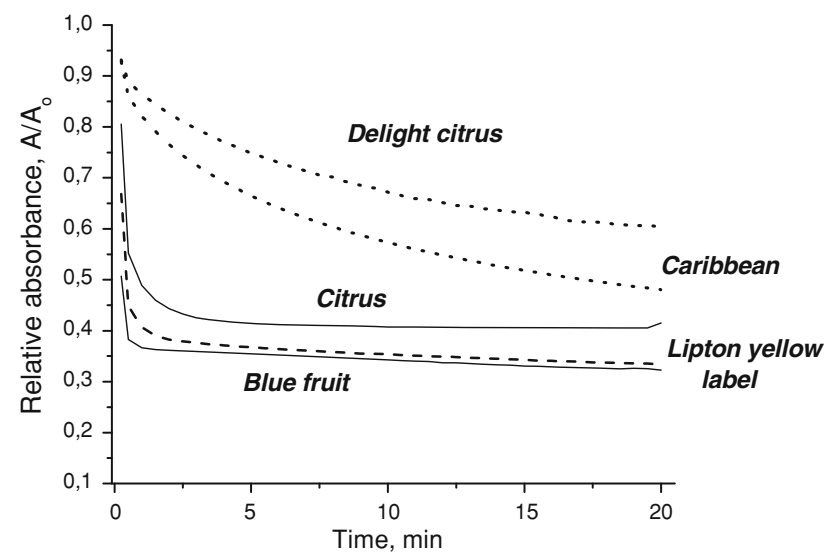

Fig. 4 The kinetic curves of scavenged DPPH by studied tea infusions. Solid lines-flavoured black teas, dotted lines-fruit teas

predominance of glycosides naringin and hesperidin. Jung et al. [24] found that supplementation with hesperidin and naringin, both citrus bioflavonoids, significantly reduced blood glucose, while the bone and lipid benefits of hesperidin make it an attractive dietary agent for the management of the health of postmenopausal women [25].

Flavoured black teas contain significant content of main catechins, particularly the concentration of EGCG in Citrus and Tropical fruit teas was high. EGCG is known to be the most active antioxidant catechin [16]. Sing et al. [14] reported that EGCG acts as an angiogenesis inhibitor by modulating protease activity during endothelial morphogenesis. Angiogenesis is a crucial step in the growth and metastasis of cancer.

The methods employed for the evaluation of antioxidant properties of studied tea infusions are based on their reduction properties (FC and CUPRAC) and the ability for scavenging of free radicals (DPPH). Conventional FolinCiocalteu method for the determination of so-called total polyphenols content is based on a non-specific phenol oxidation reaction by the two strong inorganic oxidants (phosphotungstic and phosphomolibdic acids), but this assay gives different responses to different phenolic compounds, depending on chemical structures [26]. Moreover, the FC reagent could simultaneously oxidize several nonphenolic organic compounds as well some inorganic substances to give elevated apparent phenolic content; thus, it can be used for the measurement of total reducing capacity of samples. CUPRAC assay is based on the reduction of $\mathrm{Cu}$ (II)-neocuproine complex by the combined action of all antioxidants (reducing agents) in a sample. These two methods have different reduction potentials as well as different kinetics and experimental conditions, thus do not yield the same values for the antioxidant activity. In FC assay, Yellow label tea was characterized by the highest antioxidant properties followed by Forest fruit and Tropical fruit teas, while in CUPRAC method for flavoured black teas, especially Forest fruit and tropical fruit, the highest TE values was obtained. The DPPH assay showed similar trends for tea infusion to FC and CUPRAC. Flavoured black teas were characterized by the greatest inhibition of DPPH, and the kinetic of this reaction is very fast. It can be seen that some flavoured black teas had the antioxidant properties superior to or comparable with typical black tea.

\section{Conclusion}

Evaluation of the antioxidant status of different kind of commercially available teas will promote research on the identification and quantification of active components of these teas that may help protect consumers against free radical damage and oxidative stress-related diseases. In FC and DPPH assays, the antioxidant properties of studied tea infusion increases in the order: fruit tea $<$ flavoured black tea $>$ premium black tea, while in CUPRAC method, some aromatized teas (particularly Forest fruit) exhibit the highest antioxidant properties. This is due to the phenolic components present in these tea extracts. Flavoured black teas contain significantly higher level of catechins, quercetin and rutin, while the content of chlorogenic acid, which could help lower blood sugar levels, was higher in fruit teas. Delight citrus and Caribbean fruit teas seem to be a rich source of naringin and hesperidin. The flavonone naringin has a role in the attenuation of hyperglycaemia and may exert this effect in a manner similar to the drug, metformin [27].

While higher level of phenolic antioxidants is expected to be beneficial for health, this can also lead to more astringent beverage, which may not suit all tastes. However, teas with nice smell of fruits would also support the human diet with some source of antioxidants. 
Acknowledgment The authors would like to thank the Structural Research Laboratory at the Department of Chemistry University of Warsaw for using HPLC-MS. SRL has been established with financial support from European Regional Development Found in the Programme "Improvement of the competitiveness of Enterprises" project No: WPK-1/1.4.3./1/2004/72/72/165/2005/U. The authors are thankful to Phenomenex Inc. for the donation of Kinetex column.

Open Access This article is distributed under the terms of the Creative Commons Attribution Noncommercial License which permits any noncommercial use, distribution, and reproduction in any medium, provided the original author(s) and source are credited.

\section{References}

1. Wang H, Provan GJ, Helliwell K (2000) Tea flavonoids: their functions, utilization and analysis. Trends Food Sci Technol $11: 152-160$

2. Aoshima H, Hirata S, Ayabe S (2007) Antioxidative and antihydrogen peroxide activities of various herbal teas. Food Chem 1003:617-622

3. Chen HY, Lin YC, Hsieh C (2007) Evaluation of antioxidant activity of aqueous extract of some selected nutraceutical herbs. Food Chem 104:1418-1424

4. Heaney RP (2002) Effects of caffeine on bone and the calcium economy. Food Chem Toxicol 40:1263-1270

5. Khan N, Mukhar H (2007) Tea polyphenols for health promotion. Life Sci 81:519-533

6. Sharangi AB (2009) Medicinal and therapeutic potentialities of tea (Camelia sinensis L.) - a review. Food Res Inter 42:529-535

7. Huang D, Ou B, Prior RL (2005) The chemistry behind antioxidant capacity assays. J Agri Food Chem 53:1841-1856

8. Benzie IF, Szeto YT (2002) Total antioxidant capacity of teas by the ferric reducing/antioxidant power assay. J Agri Food Chem 47:633-636

9. Kris-Etherton PM, Keen C (2002) Evidence that the antioxidant flavonoids in tea and cocoa are beneficial for cardiovascular health. Cur Opin Lipidol 13:41-49

10. Biesaga M, Pyrzynska K (2009) Liquid chromatography/tandem mass spectrometry studies of the phenolic compounds in honey. J Chromatogr A 1216:6620-6628

11. Apak R, Güçlü K, Özyürek M, Karademir SE (2004) Novel total antioxidant capacity index for dietary polyphenols and vitamins $\mathrm{C}$ and $\mathrm{E}$, using their cupric ion reducing capability in the presence of neocuproine: CUPRAC method. J Agri Food Chem 52:79707981

12. Tripoli E, La Guardia M, Giammanco S, Di Majo D, Giammanco M (2007) Citrus flavonoids: molecular structure, biological activity and nutritional properties: a review. Food Chem 104: 466-479

13. Mattila P, Hellström J, Törrönen R (2006) Phenolic acids in berries, fruits and beverages. J Agri Food Chem 54:7193-7199

14. Sing AK, Seth P, Anthony P, Husain MM, Madhavan S, Mukhtar H, Maheshwari RK (2002) Green tea constituent epigallocatechin-3-gallate inhibits angiogenic differentiation of human endothelial cells. Arch Biochem Biophys 401:29-37

15. Gattuso G, Barreca D, Gargiulli C, Leuzzzi U, Caristi C (2007) Flavonoid composition of Citrus juices. Molecules 12:1641-1673

16. Atoui AK, Mansouri A, Boskou G, Kefalas P (2005) Tea and herbal infusion: their antioxidant activity and phenolic profile. Food Chem 89:27-36

17. Peterson J, Dwyer J, Bhagwat S, Haytowitz D, Holden J, Eldridge AL et al (2005) Major flavonoids in dry tea. J Food Comp Anal 18:487-501

18. Kong JM, Chia LS, Goh NK, Chia TF, Brouillard R (2003) Analysis and biological activities of anthocyanins. Phytochemistry 64:923-933

19. Fotti MC, Daquino C, Geraci C (2004) Electron-transfer reaction of cinnamic acids and their methyl esters with the DPPH centre dot radical in alcoholic solutions. J Org Chem 69:2309-2314

20. Scharbiert S, Holzmann N, Hofmann T (2004) Identification of the astringent taste compounds in black tea infusions by combining instrumental analysis and human bioresponse. J Agri Food Chem 52:3498-3508

21. Drewnowski A, Gomez-Carneros C (2000) Bitter taste, phytonutrients, and the consumer: a review. Am J Clin Nutr 72:14241435

22. Clifford MN (2000) Chlorogenic acids and other cinnamatesnature, occurrence, dietary burden, absorption and metabolism. J Sci Food Agr 80:1033-1043

23. Lafay S, Gil-Izquierdo A, Manach C, Morand C, Besson C, Scalbert A (2006) Chlorogenic acid is absorbed in its intact form in the stomach of rats. J. Nutr 36:1192-1197

24. Jung UJ, Lee MK, Jeong KS, Choi MS (2004) The hypoglycemic effects of hesperidin and naringin are partly mediated by hepatic glucose-regulating enzymes in $\mathrm{C} 57 \mathrm{BL} / \mathrm{KsJ}-\mathrm{db} / \mathrm{db}$ mice. J Nutr 134:249-250

25. Horcajada MN, Habauzit V, Trzeciakiewicz A, Morand C, GilIzquierdo A, Mardon J, Lebecque P, Davicco MJ, Chee WS, Coxam V, Offord E (2008) Hesperidin inhibits ovariectomizedinduced osteopenia and shows differential effects on bone mass and strength in young and adult intact rats. J Appl Physiol 104:648-654

26. Moon JK, Shibamoto T (2009) Antioxidant assays for plant and food components. J Agri Food Chem 57:1655-1666

27. Purushotham A, Tian M, Belury MA (2009) The citrus fruit flavonoid naringenin suppresses hepatic glucose production from Fao hepatoma cells. Mol Nutr Food Res 53:300-307 